\title{
LITERACIA EM SAÚDE E CUIDADOS PÓS-INFARTO AGUDO DO MIOCÁRDIO
}

\author{
Vanessa Piccinin Paz ${ }^{1}$, Maria de Fátima Mantovani ${ }^{1}$, Nen Nalú Alves das Mercês ${ }^{1}$, Verónica \\ de Azevedo Mazza ${ }^{1}$ e Ângela T. Mattei da Silva ${ }^{1}$
}

1Universidade Federal do Paraná, Brasil.vanessa.piccinin7@gmail.com; mfatimamantovani@ufpr.br; nennalu@ufpr.br; mazzas@ufpr.br; angelataismattei@gmail.com

\begin{abstract}
Resumo. Introdução: O infarto do miocárdio (IAM) é uma das principais complicações cardiovasculares e requer tratamento imediato para evitar pior prognóstico. A busca pelo cuidado à saúde realizada pelas pessoas infartadas relaciona-se com a forma com que elas vivenciam e compreendem o adoecimento e o quanto este adoecer impacta na sua rotina de vida. Objetivos: Relacionar o conhecimento e o cuidado das pessoas pós IAM, com a literacia em saúde. Método: pesquisa qualitativa descritiva, realizada no período de fevereiro a maio de 2018 com 12 adultos jovens. Na coleta de dados foi utilizado um questionário semiestruturado, roteiro de entrevista e diário de campo. Para o tratamento dos dados, utilizou-se um software de pesquisa qualitativa, para posterior analise do conteúdo. Resultados: após a análise surgiram seis classes, sendo escolhido para apresentação a classe nominada como e "(Des)conhecimento e (Des)cuidado em relação a Literacia em saúde. Conclusões: A baixa literacia em saúde, está diretamente relacionada com o entendimento que as pessoas possuem sobre a sua doença e a relação com o cuidado, vindo a influenciar na percepção que elas tem sobre a gravidade da doença e consequentemente na busca pelos serviços de saúde.
\end{abstract}

Palavras-chave: Infarto Agudo do Miocárdio; Saúde do Adulto; Doença Crônica.

\section{HEALTH LITERACY AND MYOCARDIAL ACUTE POST-INFARCTION CARE}

Abstract. Introduction: Myocardial infarction (AMI) is one of the main cardiovascular complications and requires immediate treatment to avoid a worse prognosis. The search for health care carried out by infarcted people is related to the way they experience and understand the illness and how much it affects their routine of life. Objectives: To relate the knowledge and care of post-AMl people to health literacy. Method: qualitative descriptive research, carried out from February to May 2018 with 12 young adults. For data collection, a semi-structured questionnaire, interview script and field diary were used. For the treatment of the data, a qualitative research software was used, for later analysis of the content. Results: after the analysis, six classes emerged, and the class named "e (Un) knowledge and (Un) care in relation to Health Literacy was chosen for presentation. Conclusions. Low health literacy is directly related to the understanding that people have about their disease and the relationship with care, influencing their perception of the severity of the disease and, consequently, the search for health services.

Keywords: Acute Myocardial Infarction; Adult Health; Chronic disease.

\section{INTRODUÇÃO}

As doenças cardiovasculares (DCVs) são um grupo de doenças responsáveis por ocasionar 18,3 mortes a cada 100.000 pessoas, e dentre elas destaca-se o Infarto Agudo do Miocárdio (IAM) (Santos et al., 2018). O IAM é uma doença incapacitante que atinge as faixas etárias mais elevadas, porém, devido às mudanças no estilo de vida das pessoas, associadas ou não ao componente genético, essa doença também pode acometer adultos jovens em idade 
produtiva (Lima, Silva, Mendonça, Moura, \& Mattos, 2018; Goes, Barreto Filho, \& Barreto, 2016).

Essa realidade pode ser explicada pela mudança no estilo de vida das pessoas, relacionado a hábitos e comportamentos pouco saudáveis, que colaboram para o desenvolvimento de doenças como a hipertensão arterial associada ou não com o diabetes mellitus, as quais podem estar direta ou indiretamente relacionadas a episódios de IAM (Zangirolani, Assumpção, Medeiros, \& Barros, 2018).

As consequências geradas pelo IAM podem desencadear alterações físicas e psicológicas que perduram por um longo tempo após o evento agudo, fato, que leva as pessoas acometidas a refletirem sobre sua finitude, gerando sentimentos de angústia e medo (Vieira, Souza, Cavalcante, Carvalho, \& Almeida, 2017).

As mudanças ocasionadas pelo IAM interferem na qualidade de vida das pessoas acometidas influenciando na execução das suas atividades rotineiras (Passinho, Sipolatti, \& Fioresi, 2018). Embora, exista a necessidade da manutenção do controle dos fatores de risco e adesão terapêutica, observou-se na literatura que após o evento cardíaco, as pessoas apresentam resistência em dar continuidade ao tratamento no domicílio, por não aderirem ao uso terapêutico da medicação ou não manterem um estilo de vida mais saudável (Reveles, Simões, \& Ferreira, 2018).

Uma vez que a concepção de saúde das pessoas se constrói ao longo da vida, por meio das experiências e estão atreladas as crenças, ideias, valores, aspectos emocionais e na forma que buscam compreender a sua doença, a decisão para agir depende da combinação da compreensão que elas possuem em relação a sua vulnerabilidade e a sua associação sobre o entendimento da gravidade da doença, para então conseguirem perceber os benefícios obtidos diante da realização das medidas de prevenção (Coelho, Barros, Matheus, \& Domingues, 2016).

O conhecimento das alterações causadas pelo IAM pode auxiliar na redução dos fatores de riscos, na identificação precoce dos sinais e sintomas, e na busca aos serviços de saúde antes do agravamento do quadro clínico (Reveles et al., 2018). Diante disso a identificação do nível de conhecimento das pessoas com doenças crônicas e os cuidados necessários para a prevenção de complicações permite que sejam traçadas estratégias de intervenções 
educativas, com o objetivo de preencher esta lacuna, independentemente do nível de educação formal (Araújo, Jesus, Teixeira, Cunha, Santos, \& Miranda, 2018).

Ademais, a elaboração de estratégias integradas e sustentáveis de prevenção e controle da doença podem auxiliar para a melhor auto percepção da saúde e melhor enfrentamento pelos pacientes (Lima et al., 2018).

Embora as pessoas considerem o episódio agudo do infarto como um evento estressor, marcado por sentimentos de angústia e medo, estas permanecem com os mesmos hábitos que resultaram no desencadeamento do evento crítico, mediante esse fato, questiona-se: a falta de conhecimento em relação aos cuidados com a saúde após o IAM, contribui para que as pessoas mantenham comportamentos de risco para a saúde?

Diante do exposto, o objetivo deste trabalho é: Relacionar o conhecimento da doença e cuidado pós IAM com a literacia em saúde.

\section{METODOLOGIA}

Trata-se de um estudo qualitativo descritivo, derivado de uma dissertação de mestrado, que seguiu as diretrizes do "Consolidated Criteria for Reporting Qualitative Research" (COREQ). Participaram da pesquisa 12 adultos de ambos os sexos, na faixa etária de 18 a 65 anos, acometidos por IAM, no período de fevereiro a maio de 2018 , tendo como critérios de inclusão: ter diagnóstico de IAM em prontuário, sendo confirmado por familiar ou pelo participante, ser morador do município de desenvolvimento da pesquisa, ter sido atendido em uma unidade de pronto atendimento e transferido para o hospital de referência em cardiologia. Para a exclusão utilizou-se o Mini-exame do Estado Mental (Mini-Mental), tendo como ponto de corte a pontuação mínima requerida de 13 para analfabetos, 18 para baixa e média escolaridade e 26 para a alta escolaridade, consideram-se como baixa escolaridade os valores de um a quatro; média escolaridade, de quatro a oito anos incompletos; e alta escolaridade com mais de oito anos (Bertolucci, Brucki, Campacci, \& Juliano, 1994).

Para a seleção dos participantes, a pesquisadora principal verificava diariamente a lista de internamento e transferência do setor de Serviço de Arquivo Médico das Unidades de Pronto Atendimento do município. Após a identificação dos possíveis participantes pela constatação diagnóstica obtida na ficha de atendimento ambulatorial on-line, foi realizado o contato 
telefônico e agendamento de uma visita domiciliar com a finalidade de apresentar o projeto de pesquisa, em local, data e horário definido pelo participante.

Para a coleta de dados utilizou-se de um formulário semiestruturado que contemplou variáveis sociodemográficas, clínicas e os hábitos de vida dos participantes. Também se utilizou de um roteiro de entrevista com 10 questões abertas e o diário de campo, no qual foram anotados aspectos sobre o ambiente físico, interação familiar e respostas verbais e não verbais. As entrevistas foram gravadas pela pesquisadora, com o consentimento prévio dos entrevistados e tiveram duração média de uma hora.

Após a transcrição e leitura das entrevistas na íntegra, utilizou-se como apoio para a análise de conteúdo o software Interface de $R$ pourles Analyses Multidimensionnelles de Textes et de Questionnaires (IRAMUTEQ_0.6-alpha3®), para a codificação dos dados.

A caracterização dos participantes foi apresentada por meio de tabelas e analisada por meio de estatística descritiva com auxílio do Microsoft Excel® 2010. Para análise lexical, realizouse a leitura dos relatos dos participantes com vistas ao entendimento, para posterior fragmentação e construção do corpus textual, bem como para inserção no software de análise o IRAMUTEQ_0.6-alpha3®. A análise de conteúdo dos depoimentos foi realizada de acordo com as etapas propostas por Creswell (2014): 1) pré-análise, 2) exploração do material ou codificação e tratamento dos resultados e 3) interpretação.

O estudo foi submetido ao Comitê de Ética em Pesquisas no estado do Paraná e aprovado sob parecer $n^{0}$ 3.178.58. Todas as recomendações da Resolução 466/2012 do Conselho Nacional de Saúde foram atendidas. Para a realização do estudo, obteve-se o termo de autorização da Secretaria Municipal de Saúde da cidade em que se deu o estudo. Os participantes assinaram o Termo de Consentimento Livre e Esclarecido. Após a transcrição integral do conteúdo das entrevistas, uma cópia foi entregue ao participante para leitura prévia do material e a confirmação ou retificação de algum trecho lido. As informações coletadas foram mantidas em anonimato; e o nome do participante, substituído por um código identificador, com a utilização da letra EP (entrevista/participante) seguida por um número arábico em ordem crescente (EP_1, EP_2...).

A pesquisa foi desenvolvida no domicílio do participante, em local reservado, sem a presença de outras pessoas, excetuando-se os casos em que o participante o solicitou. 


\section{RESULTADOS}

As transcrições das entrevistas possibilitaram um corpus geral constituído por doze textos, separados em 226 segmentos de texto com aproveitamento de 81,42\%. O conteúdo analisado foi categorizado em seis classes, divididas em dois subcorpus.

A classe escolhida para apresentação deste trabalho foi nominada como (des)conhecimento e o (des)cuidado das pessoas pós IAM, em relação a literacia. Os participantes foram quatro mulheres e oito homens com idade variando entre 36 a 65 anos, os quais possuíam renda maior que dois salários mínimos, casados ou com união estável, com menos de três filhos. Dois participantes não eram alfabetizados. Em relação aos hábitos de vida, oito participantes, antes do IAM, faziam uso de bebida alcoólica, dois fumavam e todos não praticavam atividades físicas.

Com base nos depoimentos observou-se o desconhecimento relacionado aos sinais e sintomas do IAM, assim como a negação da gravidade do quadro clínico. No relato de EP_7 verifica-se que apesar de já ter vivenciado a experiência de um IAM, apresentou dificuldades em relacionar os sinais e sintomas do novo episódio.

Além disso, percebe-se a falta de conhecimento em relação à doença e aos cuidados necessários com a sua saúde no domicílio, pois, há relatos de medo e insegurança com a terapêutica medicamentosa após a alta, sentimentos de ansiedade e angústias por não saberem como lidar com os desafios impostos pela doença.

"A primeira ameaça de infarto, estava trabalhando, [...] nem percebi que era uma ameaça de infarto, passei mal, dor no peito, e a dor não passava" (EP_7).

"Se tivesse a ideia do que iria acontecer, teria ido antes consultar, porque é muito ruim desse jeito. Faz 90 dias que fiz a cirurgia cardíaca e ainda não sei se os medicamentos estão adequados ou o que está acontecendo [...]" (EP_5).

"não tinha noção de tanta coisa de que o meu coração tinha um lesão, achava que era só uma veia entupida. [...] vou ter agora que tomar medicamento, eu odeio tomar medicamento, mas vou ter que tomar" (EP_12).

Já no depoimento de EP_11, o infarto era visto como algo distante de sua realidade, por ser uma pessoa jovem, e quando este foi acometido pelo evento cardíaco ficou surpreso, referiu não ter noção das ações terapêuticas realizadas na instituição hospitalar, apenas fazia o que lhe era solicitado. 
"Antes do infarto, nunca tinha ficado internado, às vezes via na TV pessoas que infartaram, mas você não tinha noção de que podia acontecer comigo, então não dava importância, mudava o canal. [...] Na verdade, não me preocupei porque não tinha noção do que estava acontecendo, nem sabia o que tinha feito no hospital. Não tinha noção do que era. O médico disse que tinha 40 por cento do coração comprometido e que era uma pessoa nova, e era para me cuidar" (EP_11).

Por não saber como lidar com a situação EP_11, preferiu seguiu as orientações da equipe médica sem questioná-las ou participar do planejamento terapêutico, e assim desconhecia quais procedimentos haviam sido realizados na instituição hospitalar, tornando-se um expectador passivo, desvinculando-se da reponsabilidade na tomada das decisões a respeito do seu tratamento.

Após o evento cardíaco e o retorno ao domicílio, os participantes precisam manter alguns cuidados com a saúde, sendo estes relacionados à mudança de hábitos e ao uso de medicações contínuas. Por meio dos depoimentos verificou-se que alguns pacientes tinham dificuldades em dar continuidade ao tratamento domiciliar, como elencado no relato de EP_9, que apesar das orientações médicas e o histórico familiar, deixava de tomar a medicação prescrita para fazer uso de chás.

"Descobri a pressão alta quando tinha 45 anos e tive que tomar medicamento. Sentia muita dor de cabeça, a pressão estava alta. Minha mãe tem, então comecei a acompanhar com o médico, que orientou a tomar o medicamento certo. Não podia atrasar, porque era grave, mas muitas vezes deixava de tomar, tomava chá de chuchu e a pressão baixava” (EP_9).

Nos depoimentos de EP_7 e EP_10, constata-se que os mesmos faziam uso irregular da terapia medicamentosa, ou seja, o uso da medicação ocorria no momento que vinham a apresentar algum sinal ou sintoma de mal estar, os quais correlacionavam a experiência anterior do infarto, então retornavam ao uso da medicação.

"Saiu à consulta com o cardiologista, fiz exames e me encaminhou para investigação, foi de 2009 até 2011 e não saiu a investigação. No ano 2011, comecei a passar mal, tinha parado o medicamento, então voltei a tomar" (EP_7).

Às vezes, eu ficava quatro dias sem tomar nenhum medicamento e quando ia medir, a pressão estava alta. Acredito que tenha a ver com a alimentação salgada, e comia muito fast-food, exagerava no sal dos alimentos, gostava de linguiça no churrasco e comia uma grande quantidade de comida"(EP_10).

Além da não adesão ao tratamento medicamentoso, os hábitos alimentares permissivos persistiam mesmo após o diagnóstico da doença, vindo a agravar o quadro clínico do participante, como pode ser evidenciado no relato de EP_10. 
Nos relatos de EP_8 e EP_9, percebe-se que mesmo depois do evento cardíaco, os participantes não procuraram dar continuidade ao tratamento e não compareceram as consultas médicas, o que resultou no agravamento do quadro clínico e um novo episódio de infarto.

"tem muita gente que chega morta. Depois do infarto comecei a tomar medicamento. O segundo infarto faz 1 mês, demorei para voltar no médico. Depois do primeiro infarto não fui mais" (EP_8).

"antes do infarto a minha vida era trabalhar e fazer festa, bebia e curtia a vida, em casa gosto de tomar chá da horta agora tenho que me cuidar não gosto de médico, ir, fiquei 2 anos e não fui mais depois do primeiro infarto não voltei no médico" (EP_9).

\section{DISCUSSÃO}

Nesta pesquisa identificou-se a baixa literacia em saúde em relação aos sinais e sintomas do infarto, assim como a dificuldade em dar continuidade à terapêutica no domicílio e em manter hábitos de vida saudáveis.

A dificuldade das pessoas em interpretarem os sinais e sintomas, e a negação da gravidade do evento cardíaco, vão ao encontro com a literatura pesquisada, num estudo realizado na Turquia, com 600 pacientes no ano de 2012, no qual se observou que apesar dos sintomas apresentados, o tempo de atraso pré-hospitalar teve uma variação de 15min há 10 dias, sendo que $33,3 \%$ dos participantes não deram importância às queixas iniciais em andamento, o que pode levar ao agravamento do quadro clínico (Koc, Durna, \& Akin, 2017).

Além disso, o IAM leva as pessoas a modificarem seus hábitos de vida de forma abrupta, em função do adoecimento e das restrições imposta pela doença, pois, conforme relato dos participantes, "não se sentiam doentes", logo, a forma que compreendem o processo de adoecimento, interfere na maneira que interpretam seus sinais e sintomas (Gyberg, Björck, Nielsen, Määttä, \& Falk, 2016), consequentemente valorizando ou não as queixas iniciais.

Em estudo realizado num serviço se cardiologia, no período de 2013 a 2015, em homens que tiveram IAM, os autores perceberam que a intensa sobrecarga sensorial, imposta as pessoas no momento do IAM, gerou conflitos emocionais, em relação ao surgimento da doença, medo da morte e a preocupação com seus familiares, pois, as pessoas não estavam esperando adoecer e ter seus hábitos de vida modificados repentinamente em função do adoecimento (Vieira et al., 2017). 
A dificuldade de gerenciar a doença e lidar com suas limitações interfere no planejamento de ações para o cuidado implicando em perda da qualidade de vida (Ammouri, Kamanyires, Raddaha, Achora, \& Obeidat, 2017). Logo, a identificação dos sinais e sintomas da doença esta diretamente correlacionada com o grau de conhecimento ou da literacia em saúde, que a pessoa possui em relação ao processo saúde-doença, como fica evidenciado num estudo realizado por Lima et al. (2019), no período de maio a agosto de 2018, com 49 pacientes adultos, que procuraram avaliar o nível de conhecimento das pessoas em relação à doença arterial coronariana, verificaram que os participantes apresentaram conhecimento bom $(16,3 \%)$, aceitável $(38,7 \%)$, pouco $(26,5 \%)$ e insuficiente $(18,3 \%)$, portanto, a população estudada apresentou pouco conhecimento em relação à doença, evidenciando a necessidade de reestruturar os processos educativos voltados para esta população.

Deste modo, uma das estratégias existentes para auxiliar na identificação dos sinais e sintomas do IAM, de forma mais precoce, é a educação em saúde, ou seja, pela literacia em saúde, a qual contribui para o empoderamento das pessoas tornando-as participantes ativas do processo de cuidado, por meio da autogestão. Pois, a partir do momento em que elas conhecem suas potencialidades e limitações em relação à doença, bem como os sinais e sintomas, elas podem obter melhor qualidade de vida, vindo a ser fundamental para a promoção e manutenção do cuidado (Balsas, Silveira, Bilro, \& Marques, 2017).

Quando as pessoas compreendem à sua condição de saúde, tendem a melhorar a adesão ao tratamento e consequentemente à qualidade e estilo de vida. Portanto, pode-se enfatizar que a literacia em saúde, consiste num processo dinâmico, no qual as pessoas tornam-se participantes ativos na tomada de decisão, e passam a assumir a responsabilidade sobre a sua terapêutica (Loreiro, Mendes, Barroso, Santos, Oliveira, \& Ferreira, 2012).

Além disso, as pessoas que tem dificuldades em entender e utilizar as informações estão sujeitas, no momento em que enfrentam problemas de saúde, a terem dificuldade ou não conseguirem os gerenciar de maneira eficaz, fato que contribui para o alto índice de hospitalizações prolongadas e, onerosas para o sistema de saúde, devido à exacerbação ou complicação das doenças crônicas, como o IAM (Direção Geral da Saúde, 2016).

Em alguns relatos dos depoentes, observou-se que o IAM não foi um evento primário, já que estes, não cumpriam com as orientações relacionadas à mudança no estilo de vida, adesão medicamentosa e comparecimento às consultas médicas, vindo a desencadear um novo infarto. 
Em estudo realizado com 10 pacientes submetidos à cirurgia de revascularização do miocárdio, no Sul do Brasil, observou-se que os significados atribuídos ao processo de adoecer e a cirurgia para pacientes pós-cirúrgicos, contemplavam dificuldades de apropriação do quadro da doença e a surpresa diante da necessidade de ser submetido a um procedimento cirúrgico, assim como, tinham dúvidas em relação à severidade da situação, pois, consideravam-se jovens, portanto inatingíveis por qualquer adoecimento (Camponogara, Silveira, \& Cielo, 2014) o que também foi evidenciado nesta pesquisa.

A informação, por si só, não modifica a maneira de auto cuidar-se, já que os participantes assumem riscos, mais ou menos conscientes, que resultam na dificuldade de adesão a um estilo de vida mais saudável, e também interferem na busca pelo cuidado à saúde (Mássimo, Souza, \& Freitas, 2015).

Em estudo realizado, no Brasil, de janeiro a dezembro de 2016, com 100 pacientes diagnosticados com insuficiência cardíaca descompensada, no momento da alta hospitalar, observou-se que $11 \%$ dos pacientes apresentaram adequada literacia em saúde, sendo $21 \%$ categorizados em literacia marginal e $68 \%$ tinham literacia em saúde inadequada. Em relação à adesão medicamentosa e barreiras para a terapêutica, após 90 dias da alta hospitalar com 95 pacientes, observou-se que 41,1\% apresentaram baixa adesão medicamentosa, a qual estava associada com inadequada literacia em saúde, o que também se evidenciou em relação à reinternação e óbito, no qual pacientes com inadequada literacia em saúde apresentaram maiores percentuais (Oscalices, Okuno, Lopes, Batista, \& Campanhoro, 2019).

A não adesão medicamentosa desempenha importante papel nas complicações e agudizações das condições crônicas, resultando em atendimentos de emergência e hospitalizações (Kripalani, Goggins, Nwosu, Schildcrout, Mixon, McNaughton, Scott, \& Wallston, 2015). Fato corroborado também por um estudo realizado no Tenesse (EUA), o qual observou que $70,7 \%$ das pessoas com síndrome coronária aguda ou insuficiência cardíaca aguda, após a alta hospitalar, indicaram algum grau de não adesão aos medicamentos em períodos que antecederam a reinternação, assim como observado nesta pesquisa.

A literatura pesquisada corrobora com os dados encontrados na pesquisa, pois a baixa literacia em saúde está relacionada a problemas de entendimento por parte da pessoa acometida, em relação ao seu estado de saúde (ou falta dele), podendo ainda, estar 
associada a menor procura pelos serviços de saúde, escolhas de vida menos saudáveis, baixa adesão à terapêutica e pior entendimento sobre sua utilização, acarretando em problemas na autogestão da doença e maior índice de internação hospitalar e mortalidade (Oscalices et al., 2019), o que também foi identificado nos depoimentos, já que numa parcela dos participantes, o evento cardiológico não era mais primário.

Sendo assim, ressalta-se que a alfabetização em saúde se estende além do ambiente hospitalar onde os cuidados de saúde são prestados, pois engloba a forma como as pessoas se relacionam e a trajetória que percorrem na busca pelo autocuidado (Magnani et al., 2018), sendo está, construída por meio das experiências vivenciadas, a qual sofre influencia das crenças, valores e hábitos de vida, incorporados ao longo da sua existência, vindo a repercutir na forma pela qual elas buscam compreender a sua doença e aderir ao tratamento, assim como podem interferir na busca pelos serviços de saúde (Coelho et al., 2016).

\section{CONCLUSÕES}

Verificou-se que a baixa literacia em saúde influenciou na busca pelo cuidado com a saúde e na percepção da gravidade dos sinais e sintomas, além disso, resultou na dificuldade na adesão terapêutica.

Por meio dos depoimentos, foi possível perceber a dificuldade dos participantes em compreender que o cuidado deve ir além da agudização, sendo necessário que os profissionais de saúde, conheçam as crenças, valores e o contexto em que as pessoas estão inseridas, a fim de construir um vínculo de confiança e respeito, e estabelecer uma relação de apoio continuado fortalecendo o autocuidado, tornando-os participantes ativos do processo de cuidar.

Assim a metodologia empregada permitiu uma visão mais ampla sobre os dados encontrados, possibilitando identificar a vivência, as representações e o conhecimento das pessoas sobre a sua doença, sendo capaz de auxiliar no planejamento do cuidado e, possivelmente, na prevenção de complicações associadas, assim como melhorar a adesão ao tratamento, já que quanto maior o conhecimento em relação a sua doença, maior tende a ser a sua confiança e segurança para realizar o autocuidado. 


\section{REFERÊNCIAS}

Ammouri, A. A., Kamanyires, J.cK., Raddaha, A. H. A., Achora, S., \& Obeidat, A. A. (2017). Another Chance at life: Jordanian patients' experience of going through a myocardial infarction. Research and theory for nursing practice: An international journal, 31(4), 334-338.

Araújo, I. M. B., Jesus, A. F., Teixeira, M. L., Cunha, A. R. S., Santos, F. M. S, \& Miranda, S. R. F. (2018). Literacia em saúde de utentes com hipertensão e diabetes de uma região do norte de Portugal. Revista de Enfermagem Referência, IV(18), 73-82.

Balsas, A. M., Silveira, S. M., Bilro, A. S., \& Marques, M. C. (2017) Gestión de autocuidado del paciente con insuficiencia cardiaca: intervención de enfermería revisión integradora. Revista lbero-Americana de Saúde e Envelhecimento, 3(2), 967-980

Bertolucci, P. H. F., Brucki, S. M. D., Campacci, S. R., \& Juliano, Y. (1994). O mini-exame do estado mental em uma população geral: impacto da escolaridade. Arquivo de Neuropsiquiatria, 52(1), 1-7.

Camargo, B. V., \& Justo, A. M. (2013). IRAMUTEQ: um software gratuito para análise de dados textuais. Temas em Psicologia, 21(2), 513-518

Camponogara, S., Silveira, M., \& Cielo, C. (2014) Percepções de pacientes submetidos à cirurgia cardiovascular sobre o adoecimento. Revista de Enfermagem do Centro Oeste Mineiro, 4(1), 933-1003.

Coelho, A. C., Barros, A. L. B. L., Matheus, M. C. C., \& Domingues, T. A. M. (2016). Crenças, valores e atitudes dos familiares conviventes com pacientes coronariopatas. Revista Mineira de Enfermagem, 20, e980.

Creswell, J. W. (2014). Investigação Qualitativa: Projeto de Pesquisa escolhendo entre cinco abordagens (3rd ed.). São Paulo: Editora: Penso.

Direção Geral da Saúde. Direção de Serviços de Informação e Análise. (2016). A saúde dos portugueses. Lisboa, Portugal.

Gois, R. B. S. de. M., Filho Barreto, J. A. S., \& Barreto, R. A. (2016). Mudança de estilo de vida em situações de risco cardiovascular. Estudos de psicanálise, 45, 129-138.

Gyberg, A., Björck, L., Nielsen, S., Määttä, S., \& Falk, K. (2016). Women's help-seeking behaviour during a first acute myocardial infarction. Scandinavian Journal of Caring Sciences, 30, 670-677.

Koc, S., Durna, Z., \& Akin, S. (2017). Interpretation of symptoms as a cause of delays in patients with acute myocardial infarction, Istabul, Turkey. Eastern Mediterranean Health Journal La Revue de Santé de la Méditerranée orientale, 23(4), 287-294.

Kripalani, S., Goggins, K., Nwosu, S. Schildcrout, J., Mixon, A.S., McNaughton, C., McDougald, S., \& Wallston, K.A. (2015). Medication Non-Adherence before Hospitalization for Acute Cardiac Events. Journal of Health Communication, 20(suppl 2), 34-42.

Lima, D. M. de., Silva, D. P. da., Mendonça, I. O., Moura, N. S., \& Mattos, R. T. de. J. (2018). Fatores preditores para infarto agudo do miocárdio (IAM) em adultos jovens. Ciências Biológicas e da Saúde. 5(1):203-216.

Lima, F. M. A., Marques, A., Bocchi, S. C. M., Teixeira, T. R. F., Jamas, M. T., \& Avila, M. A. G. (2019). Conhecimento de pacientes sobre a doença arterial coronariana. Revista Mineira de Enfermagem. 23, e1264.

Loreiro, L. M. de J., Mendes, A. M. de O. C., Barroso, T. M. M. D. de A., Santos, J. C. P. dos., Oliveira, R. A., \& Ferreira, R. O. (2012). Literacia em saúde mental de adolescentes e jovens: conceitos e desafios. Revista de enfermagem Referência, 3(6), 157-166.

Magnani, J. W., Mujahid, M., Aronow, H. D., Cené, C. W., Dickson V. V., Havranek, E., Morgenstern, L. B., Paasche-Orlow, M. K., Pollak, A., \& .Willey, J. Z. (2018). Health literacy and cardiovascular disease: 
fundamental relevance to primary and secondary prevention: a scientific statement form the American Heart Association. Health Research Alliance, 136(2), e48-e74.

Mássimo, E. A. L., Souza, H. N. F., \& Freitas, M. I. F. (2015). Doenças crônicas não transmissíveis, risco e promoção da saúde: construções sociais de participantes do Vigitel. Ciência \& Saúde Coletiva, 20(3), 679766.

Oscalices, M. I. L., Okuno, M. F. P., Lopes, M. C. B. T., Batista, R. E. A., \& Campanhoro, C. R. V. (2019). Literacia em saúde e a adesão ao tratamento de pacientes com insuficiência cardíaca. Revista da Escola de Enfermagem da USP, 53, e03447.

Passinho, R. S., Sipolatti, W. G. R., Fioresi, M., \& Primo, C. C. (2018). Sinais, sintomas e complicações do infarto agudo do miocárdio. Revista de Enfermagem da UFPE, 12(1), 247-64.

Reveles, A. F., Simões, I. M. H., \& Ferreira P. A. C. (2018). Consulta de enfermagem e controle de fatores de risco cardiovasculares na pessoa após síndrome coronariana aguda. Revista de Enfermagem Referência. IV(17), 33-42.

Santos, J., Meira, K. C., Camacho, A. R., Salvador, P. T. C. O., Guimarães, R. M., Pierin, A. M. G., Simões, T. C., \& Freire, F. H. M. A. (2018). Mortalidade por infarto agudo do miocárdio no Brasil e suas regiões geográficas: analise do efeito da idade-período-coorte. Ciência \& Saúde Coletiva. 23(5), 1621-1634.

Vieira, M. B., Souza, W. S., Cavalcante, P. F., Carvalho, I. G. M., ... Almeida, R. J. (2017) Percepções de homens após infarto agudo do miocárdio. Revista Brasileira em Promoção da Saúde. 30(3), 1-9.

Zangirolani, L. T., Assumpção, D. de., Medeiros, M. A. T. de., \& Barros, M. B. (2018). Hipertensão arterial autorreferida em adultos residentes em Campinas, São Paulo, Brasil: prevalência, fatores associados e práticas de controle em estudo de base populacional. Ciência \& Saúde Coletiva, 23(4), 1221-1232. 